INDONESIAN JOURNAL OF MEDICAL SCIENCE AND PUBLIC HEALTH

2020, Vol. 1, No. 1, 20 - 27

http://dx.doi.org/10.11594/ijmp.01.01.04

Research Article

\title{
The Probiotic Role of Lactic Acid Bacteria (LAB) in Helicobacter Pyroli Gastritis
}

\author{
Arif Sabta Aji 1,2*, Ranne Balqis 2 \\ 1 Department of Nutrition, Universitas Alma Ata Yogyakarta 55183, Indonesia \\ 2 Postgraduate Biomedical Science Department, Andalas University Padang 25127, Indonesia
}

Article history:

Submission December 2019

Revised February 2020

Accepted February 2020

*Corresponding author:

E-mail: sabtaaji@almaata.ac.id

\begin{abstract}
Gastrointestinal tract infections are still common in developing countries such as Indonesia. Gastritis as a gastric health problem has a risk factor for developing into a peptic ulcer or stomach cancer. This literature review aims to discuss the role of probiotics against the incidence of gastritis caused by Helicobacter pylori infection. This article based on a literature review of the role of lactic acid bacteria probiotics in the treatment of Helicobacter Pylori infection. It was found that the probiotic role of Lactobacillus and Bifidobacterium was non-pathogenic and they were often used as probiotic mediators for $H$. Pylori eradication. Probiotics affect the gastrointestinal tract through immunologic and non-immunological responses. Utilization of probiotic intake as one of adjuvant therapy in the treatment of gastritis disea
\end{abstract}

Keywords: Probiotic, gastritis, Lactic Acid Bacteria, helicobacter pylori

\section{Introduction}

Prevalence of gastrointestinal tract infections is still quite high in developing countries such as in Indonesia [1]. The prevalence of gastritis as one of the gastric health problems has decreased significantly in the population of developed countries over the last few decades. Although chronic gastritis is still one of the pandemic infections that often, occur with the greatest risk of becoming a peptic ulcer or gastric cancer [2]. Globally, more than $50 \%$ of people have a chronic gastritis

According to WHO the incidence of gastritis in the world is around 1.8-2.1 million of the population every year, in the UK (22\%), China (31\%), Japan (14.5\%), Canada (35\%), and France $(29.5 \%)$. In Southeast Asia, around 583,635 of the total population each year, gastritis considered a trivial thing, but gastritis is the beginning of a disease that can cause a person's pain. The percentage of the incidence of gastritis in Indonesia, according to $\mathrm{WHO}$ is $40.8 \%$, and the incidence of gastritis in several regions in Indonesia is quite high with a prevalence of 274,396 cases from 238,452,952 inhabitants. Based on Indonesia's health profile in 2011, gastritis was one of the top 10 diseases in hospitalized patients in Indonesia with 30,154 cases $(4.9 \%)[3]$.

H. Pylori infection known as one of the chronic bacterial infections that are common in humans and is associated with peptic ulcer disease, gastric cancer, and primary B cell lymphoma in the stomach [4]. The presence of $H$. Pylori in saliva, dental plaque, and feces indicates that the transmission mode of this bacterial transmission spread from person to person, which is assumed the same as a mechanism for transmitting $H$. Pylori bacteria. A study found that low hygiene standards, densely populated settlements and low sanitation are ways of spreading $H$. Pylori in children and spreading the disease [5].

H. Pylori infection control carried out with antibiotic therapy as a vaccine approach. A large-scale study identifying at how preclinical 
efficacy of a number of vaccine candidates to inhibit the development of $H$. Pylori has published, although there are still very few randomized controlled trials and most of these studies have not been successful. Until now, there has not been officially an anti- $H$. Pylori vaccine. Probiotics are an alternative in preventing the spread of $H$. Pylori in the body because they contain many microorganisms, especially Lactobacillus or Bifidobacterium, which grouped based on the ability of the microorganisms to live. Later in a certain amount, they can help to control infections that used for other health benefits [6].

The benefits of probiotics in gastrointestinal disease as antibiotics had found. H. Pylori colonization in the stomach and its relationship with gastric disease make many studies that study the effects of probiotics on H. Pylori. In vitro research shows that the ability of probiotics to inhibit or kill bacteria followed by preclinical and clinical research. Research is only one part of the efficacy of probiotics against $H$. Pylori when given alone, but it increases its ability when given along with other H. Pylori eradication treatment [7].

This current literature review aims to determine the role of probiotics on health and treatment applications caused by $H$. Pylori infection.

\section{Material and Methods}

The writing of this article based on a literature study that related to the role of probiotics in human health, especially in the application of medication caused by $H$. Pylori infection.

\section{Results and Discussion \\ Helicobacter Pylori and Gastritis}

Helicobacter Pylori (H. Pylori) is a gramnegative bacterium that can survive in the stomach acid environment, compared to other pathogenic bacteria, inhibits the body's immune system of stomach inflammation to an advanced stage, and can cause gastric cancer. One of the bacteria that can neutralize or inactivate H. Pylori is Bifidobacterium and L. acidophilus. Both bacteria are natural microflora in the human digestive tract and can produce lactic acid as the main product of carbohydrate fermentation. Besides, they are able to stimulate the formation of antibodies through consumption of food sources of probiotics so that it can support the health of the digestive tract through the balance of intestinal microbiota [8].

H. Pylori has more than 20 species that have been formally established [9]. The gastric mucosa is well protected from bacterial infections. After entering the gastrointestinal tract, $H$. Pylori must survive bactericidal activity from the gastric lumen and into the mucous layer. H. Pylori is highly adapted to special conditions in the stomach. In its body, it has flagella, which allows it to form gastric epithelial colonization. In addition, these bacteria produce the urease enzyme and hydrolyses the urea to carbon dioxide and ammonia, and increase the $\mathrm{pH}$ around the bacteria. Enzyme activity is highest at low pH. H. Pylori bacteria usually cause chronic infections because of a complex balance between host factors and bacterial virulence factors. Among the several bacterial factors, one of the main factors is encouraging inflammation of Th-17, representing Peptidylprolyl cis-trans isomerase [10].

H. Pylori infection is very common in Eastern Europe, Africa and most Asian countries. In developed countries, the prevalence has been reduced and is below $10 \%$ in children and under $30 \%$ in adults. Twenty percent of infected individuals develop symptomatic gastritis, peptic ulcer or duodenum, gastric adenocarcinoma, and non-Hodgkin's stomach lymphoma. H. Pylori is also associated with iron deficiency anemia, idiopathic thrombocytopenic purpura and vitamin B12 deficiency. Several bacterial, host and environmental factors have been studied to determine the clinical outcome of $H$. Pylori infection. Among the bacterial factors, the most important virulence genes and the severity of $\mathrm{H}$. Pylori-related diseases correlate with the presence of $\operatorname{cag} A$, vacA, s1m 1 , and babA2 genotypes [11]. Recent research results support the positive effects of eradicating $H$. Pylori bacteria to prevent the occurrence of gastric cancer and regression of mucosal atrophy and intestinal metaplasia in the gastric mucosa. Precancerous detection of lesions in the stom- 
ach is recommended to be done as early as possible by detecting and eradicating $H$. Pylori bacteria [12].

\section{Probiotics}

The definition of probiotics according to the $\mathrm{FAO} / \mathrm{WHO}$ is a living microorganism which if given in sufficient quantities provides health benefits to its host. Probiotics most commonly used in humans are microorganisms belonging to the genus Bifidobacterium, Lactobacillus, Saccharomyces, and Bacillus [13]. Intestinal microbiota includes $\sim 30$ species of Bifidobacterium, 52 species of Lactobacillus, and others, such as Streptococcus and Enterococcus [14]. The most widely studied to treat and / or prevent gastrointestinal diseases are lactic acid bacteria, namely Lactobacillus and Bifidobacterium. While these species are not pathogenic, they can withstand the rough luminal environment of the gastrointestinal tract [15].

The beneficial effects of probiotics depend on the specific strain and dosage. Clinical studies of probiotics in humans develop rapidly. The beneficial effects of probiotics have been confirmed in diseases such as acute diarrhea, antibiotic-related diarrhea, gastrointestinal functional disorders, inflammatory bowel disease, etc. H. Pylori infection is also a very popular area in using probiotics as an alternative therapy to prevent the use of antibiotics that can increase the risk of antibiotic resistance [16].

The Lactobacillus species have acid-resistant abilities and are indeed present in healthy gastric microbiota. Several studies have revealed a number of beneficial effects of certain lactobacilli, such as suppression of pathogenic bacteria in the intestine and inhibition of allergic, inflammatory and neoplastic changes [14]. Some strains can even stick to gastric epithelial cells so they can stay longer than other bacteria in the stomach. This has been confirmed in a study in which there was a possibility of detecting L. reuteri strain 55730 following the gastric epithelial host cell several hours after oral administration [17]. Elliott et al., Had observed in rats with active gastric ulcer making the local microbiota altered into gram-negative bacteria including E.Coli while the Lactobacillus species experienced a significant decrease. Processed gastric mucosa Lactobacillus improves its colonization and this process is accelerated by oral lactulose administration. This study shows that normal gastric microbiota helps maintain mucosal health. In addition, oral intake of probiotics can strengthen the protective function in the gastrointestinal tract [18]. Lactobacillus is very useful in promoting gastric ulcer healing in mice, when given as an individual probiotic strain, such as Lactobacillus rhamnosus GG. increase cell proliferation to apoptotic ratio and increase epithelial cell regeneration, especially at the ulcer margins [19].

Based on the literature review conducted by Aaragon et al as much as $>13,438$ research articles on probiotics found in Pubmed and $\sim 1,422-2015$. Many reports that the effects of probiotics on the GIT are found in in vitro research study designs, experimental animal models, and healthy respondents / not healthy. The majority found was probiotic research on Irritable Bowel Syndrome. But research that looks at the relationship between probiotics and gastric ulcers is still limited. Losses that will be caused by the host that makes this study collide with physiological conditions such as acidic environment, digestive enzymes, bile acids, and mechanical stresses that reduce the survival and growth of certain probiotics. To overcome this, high doses of probiotics have been performed. Various kinds of effects caused by probiotics on the physiological condition of the body are summarized in Figure 1 below [20]. 


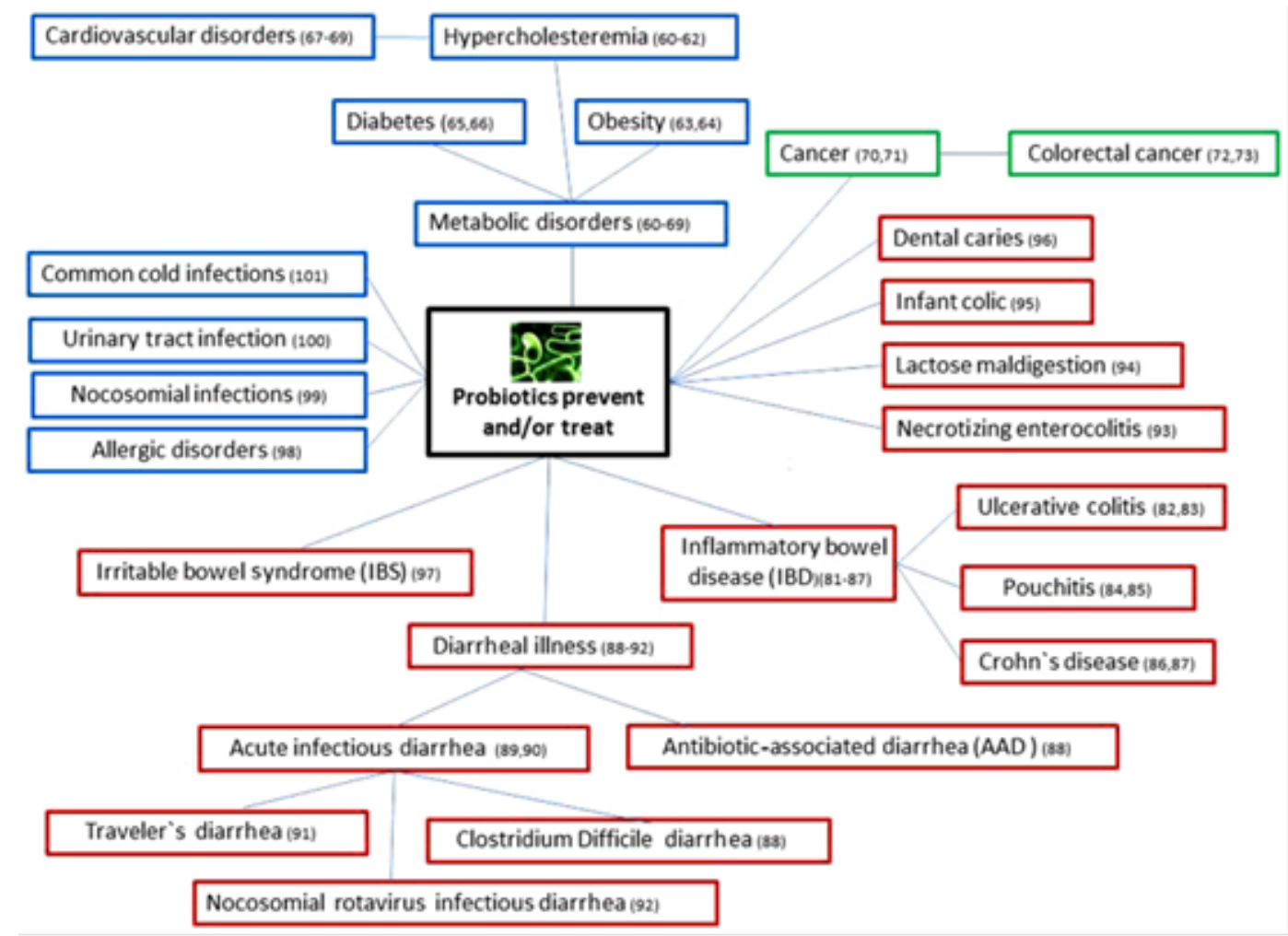

Figure 1. Summary of Gastrointestinal (red), Non-Gastrointestinal (blue) and Neoplastic (green) Disorders Known as Probiotic Response

\section{Effects of Probiotics on H. Pylori}

Stomach ulcers can occur due to stress, a bad diet, and the use of NSAIDs. The discovery of $H$. Pylori infection and its relationship with peptic ulcers is new to the practice of world gastroenterology. Typically, these bacteria are able to survive and thrive in an acidic environment so that they can cause inflammation, ulcerative, and progressive neoplastic disorders. As much as $10-20 \%$ of patients with $H$. Pylori infection can eventually cause ulcers. The regressions of recent ulcer events are highly dependent on eradicating H. Pylori [5].

Eradication of H. Pylori and changes in gastric mucosa remain a challenge for gastroenterologists. This is caused by infection with $\mathrm{H}$. Pylori which can occur early where the development of the gastric glands is characterized by prominent dividing stem cells. No antibiotic is effective enough to get rid of $H$. Pylori when given monotherapy. Triple therapy regimens (clarithromycin and amoxicillin / metronidazole combined with proton pump inhibitors) as one of the protocols in the world used in eradicating $\mathrm{H}$. Pylori. Studies using this standard therapy have shown eradication rates to reach $90 \%$. However, none of the studies has reported being able to eradicate $H$. Pylori as much as $100 \%$ [21].

Increased resistance to clarithromycin has caused a decrease in the efficiency of the triple standard therapy. To overcome this problem, new tools including quadruple, sequential, concurrent, dual and rescue therapies have been introduced. However, the development of antibiotic resistance and its side effects has caused poor patient adherence thereby limiting its application [22]. Over the past few decades, many studies have examined the possibility of using probiotics to improve H. Pylori eradication protocols and to prevent their side effects. The use of probiotics has also been tested in asymptomatic $\mathrm{H}$. pylori-infected patients and has been shown to reduce the risk of developing gastric ulcer. Kabir et al were one of the first groups that report a strain of the probiotic Lactobacil- 
lus salivarus could prevent and eliminate $H$. Pylori colonization in the stomachs of Gnotobiotic leprosy rats [23].

Based on in vitro studies using gastric epithelial cells and different probiotic strains, several effects of probiotics on $H$. Pylori infection have been identified. Probiotics can inhibit $H$. Pylori infection by non-immunological and immunological mechanisms. Non-immunological effects of probiotics include: i) Production of antimicrobials and antioxidants that can inhibit the growth activity or H. Pylori urease, ii) compete with $\mathrm{H}$. Pylori to bind the surface of gastric epithelial cells and block their specific membrane receptors, and iii) stabilize the mucosal barrier the stomach by stimulating mucus production by surface epithelial cells [24].

Probiotic immunological effects include: i) Maintaining a balance between pro and anti-inflammatory cytokines, which causes recovery from gastritis, ii) decreasing IL- 8 and TNF- $\alpha$ production by producing conjugated linoleic acid that targets the nuclear- $\mathrm{kB}$ pathway, iii) regulating regression anti-inflammatory cytokine signaling through signal transducer activation and transcription factor activator (STAT) -1 / STAT-3, and iv) increases gastric mucosal barrier by stimulating IgA secretion and transport [25].

\section{Probiotics as Alternative Therapies}

H. Pylori invasion has been considered an infection that is difficult to treat, mainly because of its resistance to commonly used antibiotics, there is a tendency for increased use of probiotics together with antibiotic regimens to eradicate H. Pylori. Probiotics have been shown to be beneficial in the treatment of several intestinal diseases such as diarrhea, besides the benefits of probiotic bacteria in the intestine; several beneficial effects on the stomach have been reported. Among them, anti-Helicobacter Pylori activity has been studied. Many therapeutic models to overcome the problem of $H$. Pylori infection in human gastrointestinal health cases ranging from allopathic therapeutic approaches which include the use of probiotics, fungi, vaccines, propolis, polysaccharides, phototherapy, microorganisms, etc [26].
The benefits of probiotic therapy in the case of $H$. Pylori are increased tolerability by preventing treatment and associated side effects. In addition, probiotics can help to correct diseases caused by H. Pylori. The clinical outcome of $H$. Pylori infection is determined by several factors, including the type of $H$. Pylori strain, degree of inflammation, and density of $H$. Pylori colonization. It has been reported that the risk of developing peptic ulcer disease and gastric cancer increases with an increased infection rate. Therefore, permanent or long-term suppression of $H$. Pylori can reduce the risk of $H$. Pylori disease [27]. Several studies have been conducted to show the beneficial effects of using probiotics against $H$. Pylori and have clarified the mechanism of action of probiotics against $\mathrm{H}$. Pylori, including its strength in mucosal inhibitors, competition for adhesion, and immunomodulatory mechanisms [28].

\section{Probiotics as a single therapy in the treatment of H. pylori}

Positive results were obtained from the first in vitro study published in 1989. Bhatia et al found that the growth of $H$. Pylori in vitro was inhibited, if L. acidophilus appeared in culture. Michetti et al reported for the first time the effects of probiotics [L. acidophilus (La1)] in $H$. Pylori colonization in humans. The study showed that the bacterial load density was reduced in the probiotic group of asymptomatic patients, whereas complete eradication of $H$. Pylori was unsuccessful. Likewise, Wang et al revealed that after intake of $B$. lactic $B b 12$ and L. acidophilus La5 in infected $H$. Pylori adults, there was a decrease in urea breath test values. Bacterial load was evaluated by the 13C-urea semi-quantitative breath test in subjects treated with L. johnsonii La1, lyophilized bacteria L.brevis CD2, B. bifidum BF-1, L. reuteri ATCC 55730, L. gasseri OLL 2716, and with probiotic multispecies such as a combination of $\mathrm{L}$. reuteri DSM 17938 and L. reuteri ATCC PTA 6457 or a combination of L. rhamnosus GG, L. rhamnosus LC705, P. Freudenreichii JS and B. lactis $\mathrm{Bb} 12$. Regardless of the probiotics used, the authors report a significant decrease in the breath test value of $13 \mathrm{C}$-urea in the probiotic 
group of patients studied $[10,29]$. In conclusion, there are only a few studies that can examine the effects of probiotics as monotherapy on the level of $\mathrm{H}$. eradication. pylori. The results showed that specific probiotics, such as S. boulardii and L. johnsonni La1 might reduce the bacterial burden, but did not completely eradicate H. Pylori bacteria.

\section{Probiotics as H. Pylori adjuvant therapy}

Several systematic reviews and Meta-analyzes of the effects of probiotics as adjuvant therapy on standard treatment of $H$. Pylori infection have been published [30]. Probiotic supplementation in general might increase the rate of eradication and reduce the frequency of side effects due to dual antibiotic therapy. However, the beneficial effects of probiotics seem to be strain specific, therefore, gathering data on different strains in a meta-analysis can lead to misleading conclusions. In this connection, a better approach is to collect data on a single probiotic strain and conduct a meta-analysis.

Szajewska et al. conducted a systematic literature review to evaluate the effect of supplementation with S. boulardii on a standard triple therapy rule at the level of $H$. Pylori eradication. Five randomized controlled trials with good methodological quality involving 1307 patients were identified. Among them, only 90 children entered. The daily dose of $\mathrm{S}$. boulardi ranges from $500 \mathrm{mg}$ to $1000 \mathrm{mg}$ and the duration of therapy is $2-4$ weeks. Of the four trials, complete data on eradication rates are available. In $80 \%$ of patients treated with S. boulardii together with triple therapy, eradication is confirmed by standard diagnostic tools [31].

\section{Probiotic Mechanism in H. Pylori}

Probiotic strains, especially Lactobacilli, have shown the opposite of being in one place with the culture of $H$. Pylori bacteria in vitro studies. Several possible mechanisms for the effects of probiotics on H. Pylori are being considered. The concentration of Lactobacilli species, as colonization of commensal bacteria in the human digestive tract reaches 0-103 / mL in normal stomach fluid. They have a longer viability in the stomach because of their resistance to acidic conditions. The possible role of normal microbiota playing in protection against gastric lesions. The total level of microorganisms in the stomach tissue of healthy mice ranged from 103 to $104 \mathrm{CFU} / \mathrm{g}$, with gram-negative bacteria representing $5 \%$ of the total population. However, they showed that a few days after treatment of gastric ulcers in healthy mice, the total bacterial count peaked at 109-1010 CFU / g and maintained this status for about 7 days. At present, gram-negative microorganisms comprise the majority of the total number of bacteria present compared to the population of lactobacilli, a reduction in colonization of gram-negative bacteria occurring everywhere in the lumen, but this event occurs at a higher rate to the ulcer side. This finding concludes that the intestinal microbiota might be the first line of defense against pathogenic bacteria. Therefore, consuming exogenous lactic acid bacteria with probiotic properties can increase this protective effect in the stomach maintaining native microbiological homeostasis, damaging the stability of $\mathrm{H}$. Pylori and decreasing the inflammatory response. This situation is certainly present in populations infected with $H$. Pylori and results in a reduction in the bacterial inflammatory response [32].

Two main types of substances including short chain fatty acids (SCFAs) and bacteriocin have been involved in the inhibition of $\mathrm{H}$. pylori. During carbohydrate metabolism, SCFAs such as aseptic, propionic, butyric, and lactic acid are secreted by probiotic organisms, and have an important role in reducing $\mathrm{pH}$. Observation of the antagonistic effect of the lactobacillus strain on $H$. Pylori involves SCFA in this effect. SCFA is believed to be an agent that mediates the effects of the antagonists. The antimicrobial effect of lactic acid has an impact on decreasing $\mathrm{pH}$, it can inhibit $H$. Pylori urease, although the reciprocal mechanism of lactobacillus against $H$. Pylori differs from strain to strain. Furthermore, certain genera of lactobacilli produce antimicrobial metabolites that are similar to those produced by the bacteriocin family. They are compounds with anti-bacterial potential of $H$. Pylori and bacteriocin which have a heat-resistant peptide structure with antimicrobial properties [33]. H. Pylori adhesion inhibition is another important mechanism that protects the gastroduodenal lumen from inflammation as well as atrophic gastritis. 
H. Pylori adhesion in host cells is important in determining disease outcome. Some Lactobacilli strains can exert their anti-adhesive activity to secrete antimicrobial substances. Therefore, Lactobacillus reuteri (L. reuteri) can inhibit the growth of $H$. Pylori by competing adhesion receptors. An important mechanism of probiotic agents is to modulate and mediate immune responses to pathogens. The presence of $H$. Pylori inflammatory response by the release of inflammatory mediators such as cytokines and chemokines can be prevented by probiotics that modulate the host immune response through interactions with epithelial cells. Reduction in gastric inflammatory activity occurs after changes in the release of antiinflammatory effectors [34]. However, the effects of probiotics on immune responses are difficult to simplify because certain probiotic strains can produce diverse immunity, which varies depending on the host's immune status and indeed the genetic variation of us might have influenced in a certain phenotypic outcomes[35-38].

\section{Conclusion}

Probiotics in general have an important role in preventing $H$. Pylori infection through the action of lactic acid bacteria. In addition, this type of probiotics can be an alternative therapy to reduce the risk of antibiotic resistance in the treatment of gastritis. Although, it still requires a lot of in-depth research and is still a debate among experts to find out the complete mechanism and the proper dosage of use. Lactic Acid Bacteria (LAB) Lactobacillus and Bifidobacterium are non-pathogenic species and are the most often used as probiotic mediators for eradication of $H$. Pylori bacteria. Probiotics work and affect the health of the digestive tract through immunological and nonimmunological responses. Utilization of probiotic intake is an adjuvant therapy in the treatment of gastritis to help the effectiveness of antibiotic drugs.

\section{Acknowledgment}

This research was funded by Directorate General of Higher Education Ministry of National Education (Menristekdikti), Indonesia, with project name The Research of Master Program Leading to Doctoral Degree for Excellent Students (PMDSU Batch-2) in the year of 2018 (Grant No: 050/SP2HL/LT/DRPM/2018) Therefore, we are grateful for this funding and support of this research.

\section{References}

1. Tumonggor MK, Karafet TM, Hallmark B, Lansing JS, Sudoyo H, Hammer MF, et al. (2015) The Indonesian archipelago: an ancient genetic highway linking Asia and the Pacific. J Hum Genet. 58(3):165-73.

2. Sipponen P, Maaroos H-I (2015) Chronic gastritis. Scand J Gastroenterol 50(6):657-67.

3. Kementrian Kesehatan Republik Indonesia (2011) Profil Kesehatan Indonesia 2011. Jakarta: Kementrian Kesehatan RI.

4. Peek RM, Blaser MJ (2002) Helicobacter pylori and gastrointestinal tract adenocarcinomas. Nat Rev Cancer 2(1):28-37.

5. Mhaskar RS, Ricardo I, Azliyati A, Laxminarayan R, Amol B, Santosh W, et al (2013) Assessment of risk factors of helicobacter pylori infection and peptic ulcer disease. J Glob Infect Dis 5(2):60-7.

6. Behnsen J, Deriu E, Sassone-Corsi M, Raffatellu M (2013) Probiotics: properties, examples, and specific applications. Cold Spring Harb Perspect Med. 3(3): a010074.

7. Patel A, Shah N, Prajapati JB (2014) Clinical application of probiotics in the treatment of Helicobacter pylori infection-a brief review. J Microbiol Immunol Infect Wei Mian Yu Gan Ran Za Zhi 47(5):429-37.

8. Gisbert JP, McNicholl AG (2017) Optimization strategies aimed to increase the efficacy of $H$. Pylori eradication therapies. Helicobacter 22(4): 1-9.

9. Solnick JV, Schauer DB (2001) Emergence of Diverse Helicobacter Species in the Pathogenesis of Gastric and Enterohepatic Diseases. Clin Microbiol Rev. 14(1):59-97.

10. Homan M, Orel R (2015) Are probiotics useful in Helicobacter pylori eradication? World J Gastroenterol WJG. 21(37):10644-53.

11. Homan M, Šterbenc A, Kocjan BJ, Luzar B, Zidar N, Orel R, et al. (2014) Prevalence of the Helicobacter pylori babA2 gene and correlation with the degree of gastritis in infected Slovenian children. Antonie Van Leeuwenhoek 106(4):637-45.

12. Kodama M, Murakami K, Okimoto T, Abe T, Nakagawa Y, Mizukami K, et al. (2012) Helicobacter pylori eradication improves gastric atrophy and intestinal metaplasia in longterm observation. Digestion 85(2):126-30.

13. Shahverdi E (2016) Probiotics and Gastrointestinal Diseases. Glob J Dig Dis [Internet] [cited 2017 Nov 3];2(1). Available 
from: http://digestive-diseases.imedpub.com/abstract/probiotics-and-gastrointestinal-diseases-8624.html

14. Khoder G, Al-Menhali AA, Al-Yassir F, Karam SM (2016) Potential role of probiotics in the management of gastric ulcer. Exp Ther Med. 12(1):3-17.

15. Wallace TC, Guarner F, Madsen K, Cabana MD, Gibson G, Hentges E, et al. (2011) Human gut microbiota and its relationship to health and disease. Nutr Rev. 69(7):392-403.

16. Ouwehand AC, Forssten S, Hibberd AA, Lyra A, Stahl B (2016) Probiotic approach to prevent antibiotic resistance. Ann Med. 48(4):246-55.

17. Valeur N, Engel P, Carbajal N, Connolly E, Ladefoged K (2004) Colonization and Immunomodulation by Lactobacillus reuteri ATCC 55730 in the Human Gastrointestinal Tract. Appl Environ Microbiol 70(2):1176-81.

18. Yang Y-J, Chuang C-C, Yang H-B, Lu C-C, Sheu B-S (2012) Lactobacillus acidophilus ameliorates $\mathrm{H}$. pylori-induced gastric inflammation by inactivating the Smad7 and NFkB pathways. BMC Microbiol 12:38.

19. Lam EKY, Yu L, Wong HPS, Wu WKK, Shin VY, Tai EKK, et al. (2007) Probiotic Lactobacillus rhamnosus GG enhances gastric ulcer healing in rats. Eur J Pharmacol. 565(1-3):171-9.

20. Aragon G, Graham DB, Borum M, Doman DB (2010) Probiotic Therapy for Irritable Bowel Syndrome. Gastroenterol Hepatol 6(1):39-44.

21. Malfertheiner P, Selgrad M, Bornschein J (2012) Helicobacter pylori: clinical management. Curr Opin Gastroenterol. 28(6):608-14.

22. Giorgio F, Principi M, De Francesco V, Zullo A, Losurdo G, Di Leo A, et al. (2013) Primary clarithromycin resistance to Helicobacter pylori: Is this the main reason for triple therapy failure? World J Gastrointest Pathophysiol 15;4(3):43-6.

23. Kabir AM, Aiba Y, Takagi A, Kamiya S, Miwa T, Koga Y (1997) Prevention of Helicobacter pylori infection by lactobacilli in a gnotobiotic murine model. Gut. 41(1):49-55.

24. Mack DR, Ahrne S, Hyde L, Wei S, Hollingsworth MA (2003) Extracellular MUC3 mucin secretion follows adherence of Lactobacillus strains to intestinal epithelial cells in vitro. Gut. 52(6):827-33.

25. Yang Y-J, Sheu B-S (2012) Probiotics-containing yogurts suppress Helicobacter pylori load and modify immune response and intestinal microbiota in the Helicobacter pylori-infected children. Helicobacter 17(4):297-304.

26. Aiba Y, Nakano Y, Koga Y, Takahashi K, Komatsu Y (2015) A highly acid-resistant novel strain of Lactobacillus johnsonii No. 1088 has antibacterial activity, including that against Helicobacter pylori, and inhibits gastrin-mediated acid production in mice. Microbiology Open 4(3):465-74.

27. Yang J-C, Lu C-W, Lin C-J (2014) Treatment of Helicobacter pylori infection: current status and future concepts. World J Gastroenterol 20(18):5283-93.
28. Papastergiou V, Georgopoulos SD, Karatapanis S (2014) Treatment of Helicobacter pylori infection: Meeting the challenge of antimicrobial resistance. World J Gastroenterol WJG 20(29):9898-911.

29. Francavilla R, Polimeno L, Demichina A, Maurogiovanni G, Principi B, Scaccianoce G, et al. (2014) Lactobacillus reuteri strain combination in Helicobacter pylori infection: a randomized, double-blind, placebo-controlled study. J Clin Gastroenterol 48(5):407-13.

30. Zhu R, Chen K, Zheng Y-Y, Zhang H-W, Wang J-S, Xia Y-J, et al. (2014) Meta-analysis of the efficacy of probiotics in Helicobacter pylori eradication therapy. World J Gastroenterol WJ 20(47):18013-21.

31. Szajewska H, Horvath A, Piwowarczyk A (2010) Meta-analysis: the effects of Saccharomyces boulardii supplementation on Helicobacter pylori eradication rates and side effects during treatment. Aliment Pharmacol Ther. 32(9):1069-79.

32. Kafshdooz T, Akbarzadeh A, Majdi Seghinsara A, Pourhassan M, Nasrabadi HT, Milani M (2017) Role of Probiotics in Managing of Helicobacter Pylori Infection: A Review. Drug Res. 67(2):88-93.

33. Marshall VM (2007) Probiotics and Prebiotics: Scientific Aspects (2005). Int J Dairy Technol. 60(1):63-4.

34. Dadashzadeh K, Milani M, Rahmati M, Akbarzadeh A (2014) Real-time PCR detection of 16S rRNA novel mutations associated with Helicobacter pylori tetracycline resistance in Iran. Asian Pac J Cancer Prev APJCP 15(20):8883-6.

35. Lee JH, Jun SH, Kim J-M, Baik SC, Lee JC (2015) Morphological changes in human gastric epithelial cells induced by nuclear targeting of Helicobacter pylori urease subunit A. J Microbiol Seoul Korea 53(6):406-14.

36. Aji, A.S., Erwinda, E., Rasyid, R. et al (2020). A genetic approach to study the relationship between maternal Vitamin D status and newborn anthropometry measurements: the Vitamin D pregnant mother (VDPM) cohort study. J Diabetes Metab Disord 19, 91-103.

37. Surendran S, Aji AS, Ariyasra U, Sari SR, Malik SG, Tasrif N, et al (2019). A nutrigenetic approach for investigating the relationship between vitamin B12 status and metabolic traits in Indonesian women. J Diabetes Metab Disord. 1;18(2):38999.

38. Alathari BE, Aji AS, Kalpana CA, Vimaleswaran KS (2020). Vitamin D pathway-related gene polymorphisms and their association with metabolic diseases: A literature review. J Diabetes Metab Disord. 